\title{
The critical illness mortality inflection point during prolonged Surgical ICU length of stay
}

Niels D Martin ${ }^{1 *}$, Tara Ramaswamy ${ }^{1}$, Emily Moin ${ }^{1}$, Joshua A Marks ${ }^{1}$, Tara Collins ${ }^{1}$, Nina O’Connor ${ }^{2}$, Daniel N Holena ${ }^{1}$, Jose Pascual ${ }^{1}$, Patrick M Reilly ${ }^{1}$ and Lewis J Kaplan ${ }^{1}$

${ }^{1}$ Division of Traumatology, Surgical Critical Care \& Emergency Surgery, Department of Surgery, Perelman School of Medicine at the University of Pennsylvania, USA ${ }^{2}$ Palliative Care, Department of Medicine, Perelman School of Medicine at the University of Pennsylvania, USA

\begin{abstract}
Background: The purpose of this study is to determine the relationship between ICU Length of Stay (LOS) and mortality in a surgical population and evaluate predictors of mortality at a LOS inflection point.

Methods: All patients admitted to a surgical ICU from July 2003 through June 2014 at a well-established, urban, academic medical center were retrospectively reviewed via a prospective registry. In-hospital mortality rates were determined and plotted for each ICU LOS day through the first 6 weeks. Identified mortality curve slope change was used to identify an at-risk cohort. Using the most recent one-year data, risk factors including APACHE II were recalculated on the inflection point day to assess metric validity.

Results: 19,061 ICU admissions were assessed. Mortality followed a sigmoid curve with a significant rise occurring during week two that plateaued by ICU day 14 . Mortality rates based on LOS $\leq 14$ days vs. > 14 days significantly differed ( $4.23 \%$ vs. $21.6 \%$, $p<0.001)$. There were 130 patients with a $>14$-day LOS in the most recent year cohort. Re-calculated APACHE II scores on ICU day 14 were significantly predictive of mortality, 23.64 for deaths (95\% CI: 21.36-25.92) vs. 16.35 for survivors (95\% CI: $15.50-17.20)$, p < 0.001. Additional independent day 14 mortality predictors included pressor use (44.0\% vs. $21.9 \%$, $\mathrm{p}<0.02)$, bacteremia $(44.0 \%$ vs. $16.2 \%, \mathrm{p}<0.002)$, active malignancy $(40.0 \%$ vs. $16.2 \%, \mathrm{p}<0.005)$, and dialysis ( $36.0 \%$ vs. $10.5 \%, \mathrm{p}<0.001)$.
\end{abstract}

Conclusions: Mortality significantly increases after 14 days in a surgical ICU. Recalculation of APACHE II score at this inflection point is valid and correlative of mortality. This methodology can be helpful for informing decision making for patients who require ICU care exceeding 14 days duration.

\section{Background}

Early recognition of anticipatable mortality in the Intensive Care Unit (ICU) can allow for more timely patient centered palliative care. This can then mitigate patient, family, and health care provider physical and emotional pain and anguish, and allow for leveraged resources toward comfort in a timely fashion. Prolonged Length of Stay (LOS) and chronic critical illness have long been considered predictors of long term mortality in the ICU [1-5]. Identifying patients at risk for mortality based on LOS can provide an opportunity to improve prognostication, value, and quality of care in the ICU for patients, families, and providers.

Defining a prolonged ICU LOS, however, is difficult as many factors impact the ultimate outcome. Physiologic reserve, number of failed organ systems, and patient and family values all drive management over time [6]. Further, the severity of illness necessitating ICU admission varies greatly by institution and diagnosis. For these reasons, the current literature defines a prolonged ICU LOS within a very broad range, from as short as 5 days to as high as 60 days in various populations $[2,4,6-12]$. These highly variable findings were often chosen arbitrarily and suggest that local outcomes must be used to determine a locally relevant definition of a prolonged ICU stay.

Surgical ICU patients pose additional challenges to outcome assessment and management in that most have both a critical care team and a primary surgical team involved in a shared decision-making model. This adds an additional layer of teamwork and communication before definitive management plans can be devised and implemented, even in the face of failing medical post-operative care. Moreover, the surgical team's upfront investment in the pre-, intra-, and postoperative care may render it difficult to recognize and act even when medical therapy failure is quite apparent.

The interplay between diagnoses, prolonged LOS, ultimate outcome including mortality, and the unique surgical population has yet to be extensively studied. We hypothesize that local ICU data can be used to define a prolonged LOS relevant to its own population. Recognizing that most tools for prognostication such as Acute Physiological and Chronic Health Evaluation (APACHE) were defined for shorter length of stays ${ }^{10}$, we further hypothesize that in defining a prolonged LOS time-point and its subsequent population, we can validate common predictors of mortality (including APACHE) even in this select and high-risk cohort.

Correspondence to: Niels D Martin, Division of Traumatology, Surgical Critical Care, \& Emergency Surgery Perelman School of Medicine at the University of Pennsylvania, 51 North 39th ST, Medical Office Building Suite 120, Philadelphia, PA 19104, USA, Tel: (215) 662-7323, Fax. (215) 349-5917, E-mail: Niels.Martin@ uphs.upenn.edu

Key words: ICU outcomes, ICU mortality, end of life, surgical futility, ICU length of stay

Received: December 15, 2017; Accepted: January 10, 2018; Published: January 16,2018 


\section{Methods}

This study was performed at an urban, academic medical center with a long-established surgical critical care service ( $>15$ years). The Surgical ICU functions in a semi-closed fashion, in that patients remain on the primary surgical service, but all orders are placed by the critical care service. The patient population includes adult surgical oncology, vascular, organ transplant, trauma (Level one trauma center), urology, and emergency general surgery. This ICU does not cover cardiac or isolated neurologic disorders. This study was performed with approval from the university's Institutional Review Board with a waiver of informed consent.

Data was collected via a prospectively entered surgical critical care database from 2003 through 2014 and was retrospectively reviewed for this study. Standard demographical data along with ICU admission APACHE II score, length of stay, and mortality were extracted. Inhospital mortality rates were calculated for each ICU LOS day through the first 6 weeks. A five-point moving average regression line was superimposed onto the scatterplot of mortality rates. Notable mortality curve slope change (inflection point) was then used to identify an atrisk cohort based upon length of stay related mortality.

Using the most contemporary year's data (July 2013-June 2014), this cohort was re-assessed by recalculating APACHE II scores on the slope change day (the inflection point day) identified by data from the entire cohort. Other binary variables common in the end-of-life literature were also collected on that day including prior ICU admissions in the past 30 days, ventilator-dependence, delirium, vasopressor infusion, Total Parenteral Nutrition (TPN), Total Enteral Nutrition (TEN), active malignancy, dialysis, steroids, history of alcohol or illicit drug abuse, antibiotic therapy, bacteremia, anemia, and DVT prophylaxis. These data points were only abstracted if documented on the inflection point day.

Delirium was captured in the database prospectively when the Confusion Assessment Method for the ICU (CAM ICU) score was positive for greater than 24 hours as documented in the nursing flow sheet. Anemia was defined as a hemoglobin less than the lower limit of our institutional normal, which included gradations by age and gender. The remaining variables were abstracted from the SICU Attending's daily progress note on the slope change day. A single data collector who was blinded to patient outcome, performed the APACHE II calculations and abstracted the binary variables from the medical record.

Statistical analysis was performed using SPSS (version 22). All data underwent descriptive statistical analysis yielding frequency scores for categorical data and measures of central tendency (mean \pm SD) for continuous/interval data. Statistically significant differences between variables was determined by the $t$-test for independent samples and a $z$-test of proportions to determine if patient outcome significantly varied by recalculated APACHE II scores or by any other slope change day predictor of mortality. The area under the Receiver Operating Characteristic (ROC) curves were generated for both the predictive value of admitting APACHE toward mortality and that of the day 14 recalculated APACHE mortality prediction. The Hosmer-Lemeshow statistic will be used to note acceptable calibration of the curve. A p-value $\leq 0.05$ was considered statistically significant.

\section{Results}

During the initial study period of 11 years, there were 19,061 unique patient admissions to the SICU. Re-admissions within 30 days were grouped together as one occurrence. The average length of stay was 4.14 days, though the median was 2 days. Those with stays greater than six weeks accounted for $1 \%$ of admissions but constituted $15.7 \%$ of total ICU days.

\section{Model creation cohort}

After sorting by LOS, patients were grouped by individual LOS day, and in-hospital mortality was calculated for each group (LOS day). Mortality rates for each LOS day were then plotted over time from admission through day 42 (Figure 1). A five-point moving average regression line was superimposed onto the scatterplot revealing a major rise during the second week, an inflection point at day 14 , and a generalized plateau thereafter.

Overall in-hospital mortality during the study period was $4.88 \%$. 1,153 patients had an ICU LOS > 14 day, representing approximately $6.0 \%$ of all admissions (Figure $2 \mathrm{~A}$ ). Mortality rates before and after the 14-day breakpoint differed significantly at $4.05 \%$ and $17.79 \%$ respectively, $\mathrm{p}<0.0001$ (Figure 3 ). In the $>14$-day cohort $(\mathrm{n}=130)$, the mean length of stay was 30.61 days (median 25 days) with a SD of 19.3 and a near linear distribution.

\section{Model validation cohort}

Using the most contemporary year's data (2014), similar data for LOS and in-hospital mortality were noted (Figure 2B). Using the inflection point day as a cut off, 130 patients had an ICU LOS $>14$ day in FY2014. Recalculated APACHE II scores for this cohort on ICU day 14 were significantly predictive of mortality [23.64 in non-survivors ( $\mathrm{SE}=1.10,95 \% \mathrm{CI} 21.36-25.92)$ vs. 16.35 in survivors ( $\mathrm{SE}=0.43,95 \% \mathrm{CI}$ 15.50-17.20), $\mathrm{p}<0.0001$ ] (Figure 4).

The improvement in the predictive value of the day 14 recalculated APACHE II score was performed by direct comparison of the same cohort's initial APACHE II scores on ICU admission. Using the mean APACHE II scores as the cutoff for prediction of mortality, the recalculated day 14 vs. initial APACHE II scores had an increased sensitivity ( $56 \%$ to $84 \%, \mathrm{p}=0.062)$ and increased specificity $(46.6 \%$ to $60.2 \%, \mathrm{p}=0.069)$. Positive predictive value increased from $20.3 \%$ to $33.9 \%(\mathrm{p}=0.113)$ and negative predictive value increased from $81.4 \%$ to $93.9 \%(p=0.051)$. The area under the ROC curves for APACHE prediction of mortality demonstrated improved predictive potential with the area improving from 0.5935 to 0.7554 and a HosmerLemeshow statistic of 0.73 noting acceptable calibration of the day 14 data (Figure 5).

Several additional ICU days 14 findings were similarly significantly associated with mortality. These included vasopressor infusion $(44.0 \%$ in non-survivors vs. $21.9 \%$ in survivors, $\mathrm{p}<0.02$ ), bacteremia ( $44.0 \%$ vs. $16.2 \%, \mathrm{p}<0.002)$, active malignancy ( $40.0 \%$ vs. $16.2 \%, \mathrm{p}<0.005)$, steroid administration $(40.0 \%$ vs $15.0 \%, \mathrm{p}<0.002)$, TPN infusion $(64.0 \%$ vs $38.1 \%, \mathrm{p}<0.01)$, TEN administration $(32.0 \%$ vs $59.0 \%, \mathrm{p}<0.01)$ and dialysis utilization $(36.0 \%$ vs. $10.5 \%, \mathrm{p}<0.001)$ (Figure 6$)$. Of note, the use of TEN was protective. Also, no difference in mortality was found for ventilator-dependence, delirium, history of alcohol or illicit drug abuse, antibiotic therapy, anemia, and DVT prophylaxis utilization.

\section{Discussion}

Timely recognition of anticipatable mortality in the ICU is increasingly pertinent as modern care seems able to substantially prolong indicators of life that may be quite divorced from quality of life. The goal of mortality risk prognostication would therefore be to identify patients at highest risk of mortality regardless of intervention and to offer alternatives to the provided care, including most notably, 


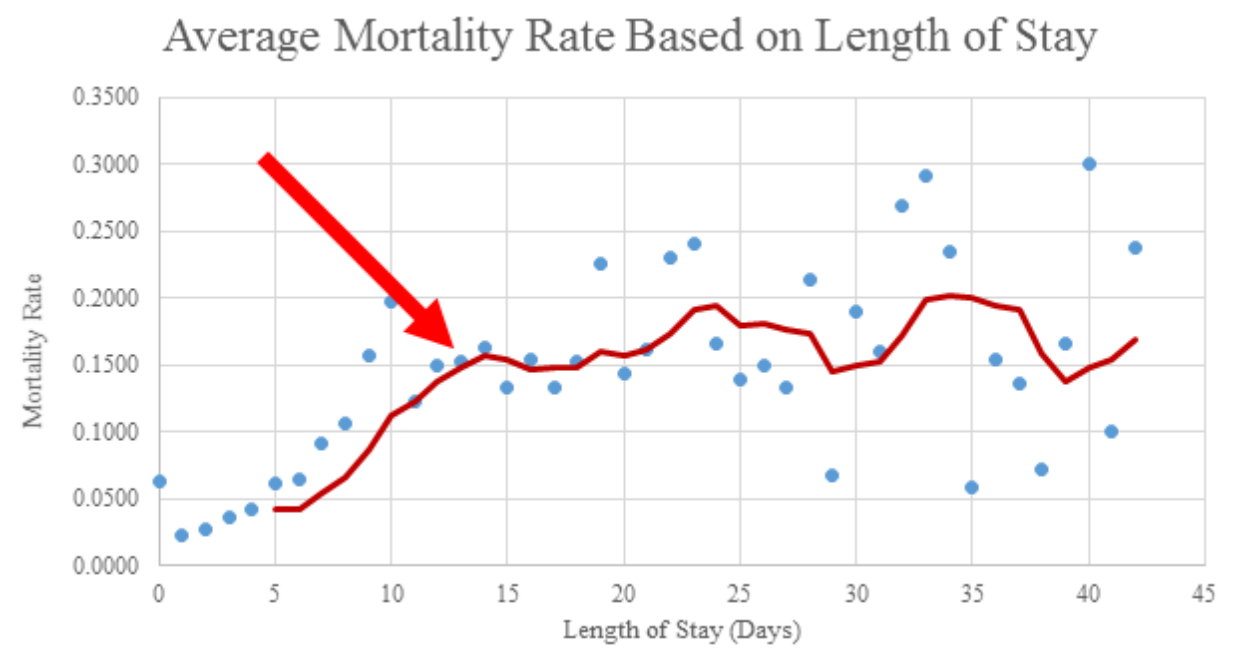

Figure 1. Scatter plot demonstrating the relationship between ICU Length of Stay and Mortality for that Length of Stay. Overlying is a 5-point moving average regression line. Heavy Arrow denotes the infection point at day 14 .

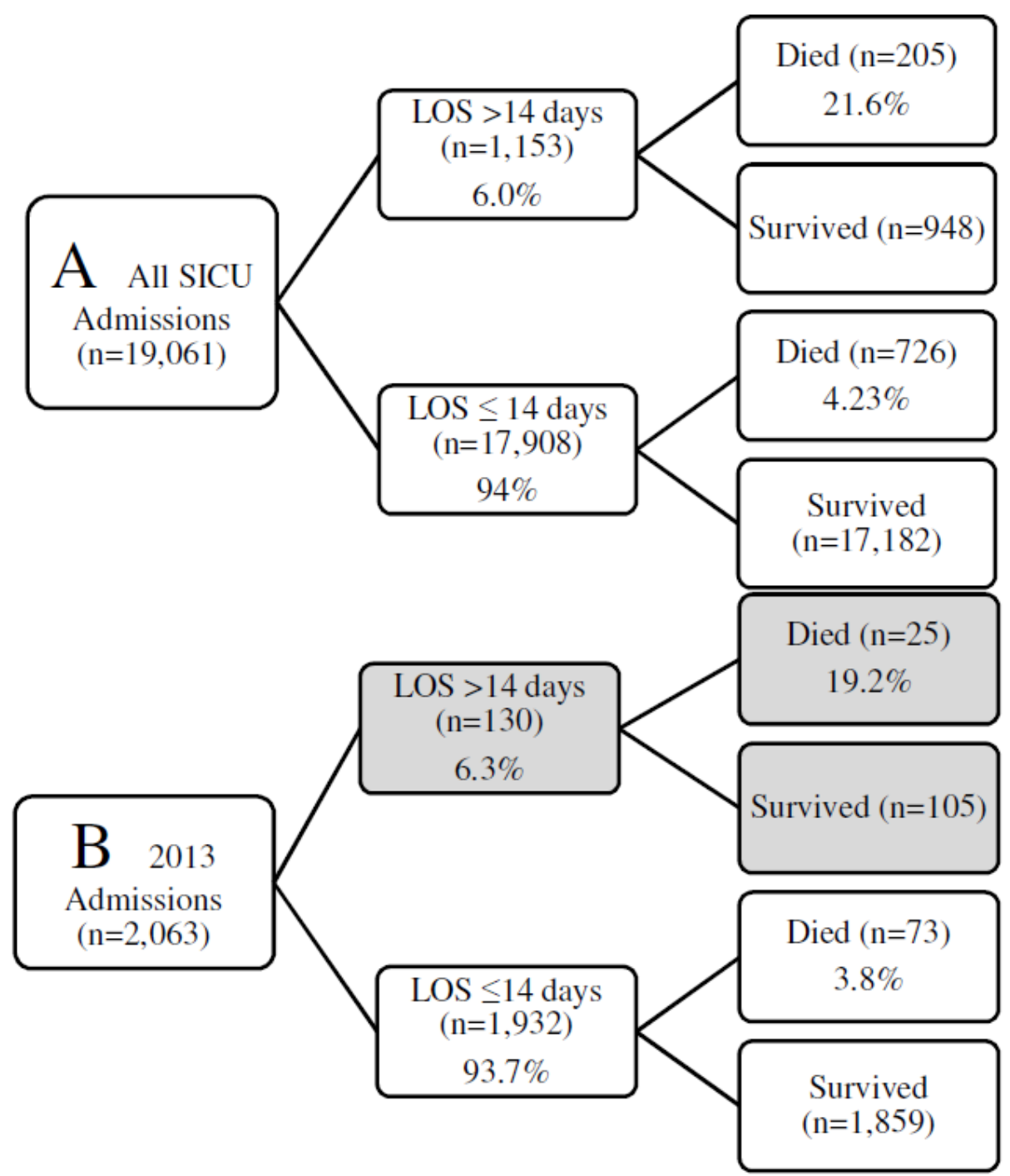

Figure 2. "A" depicts the 11 year cohort broken down by Length of Stay and Mortality. "B" depicts the most contemporary one year cohort (2014) broken down by Length of Stay and Mortality. 


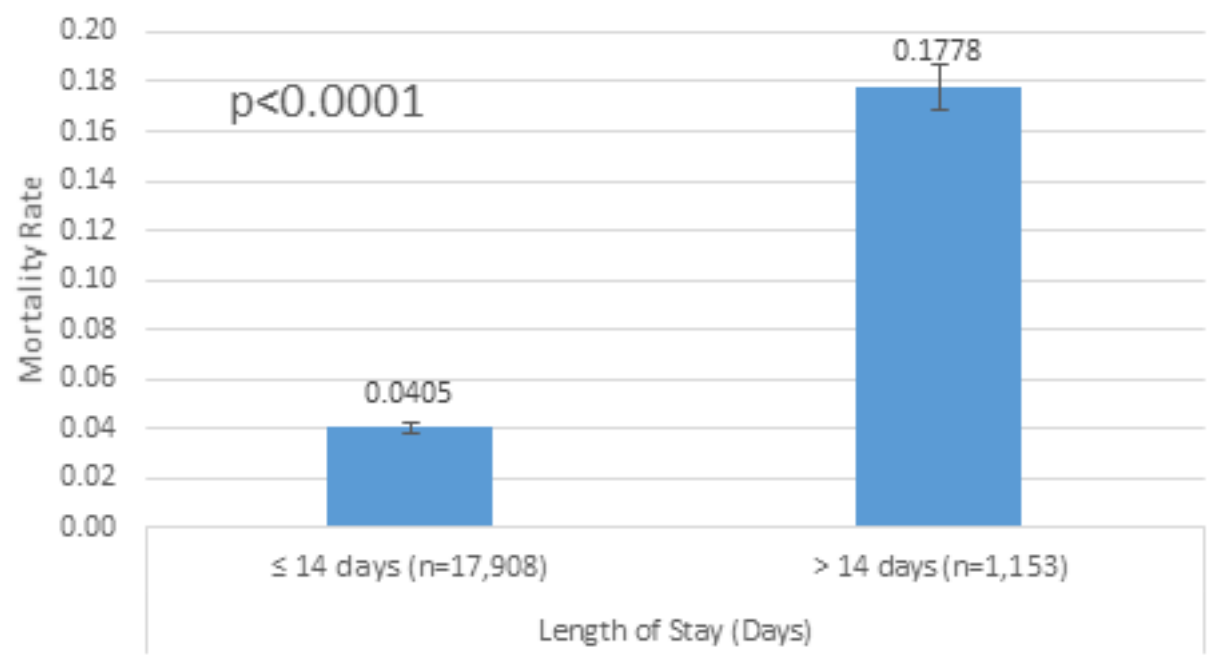

Figure 3. Mortality rate differences between Lengths of Stay shorter and longer than 14 days.

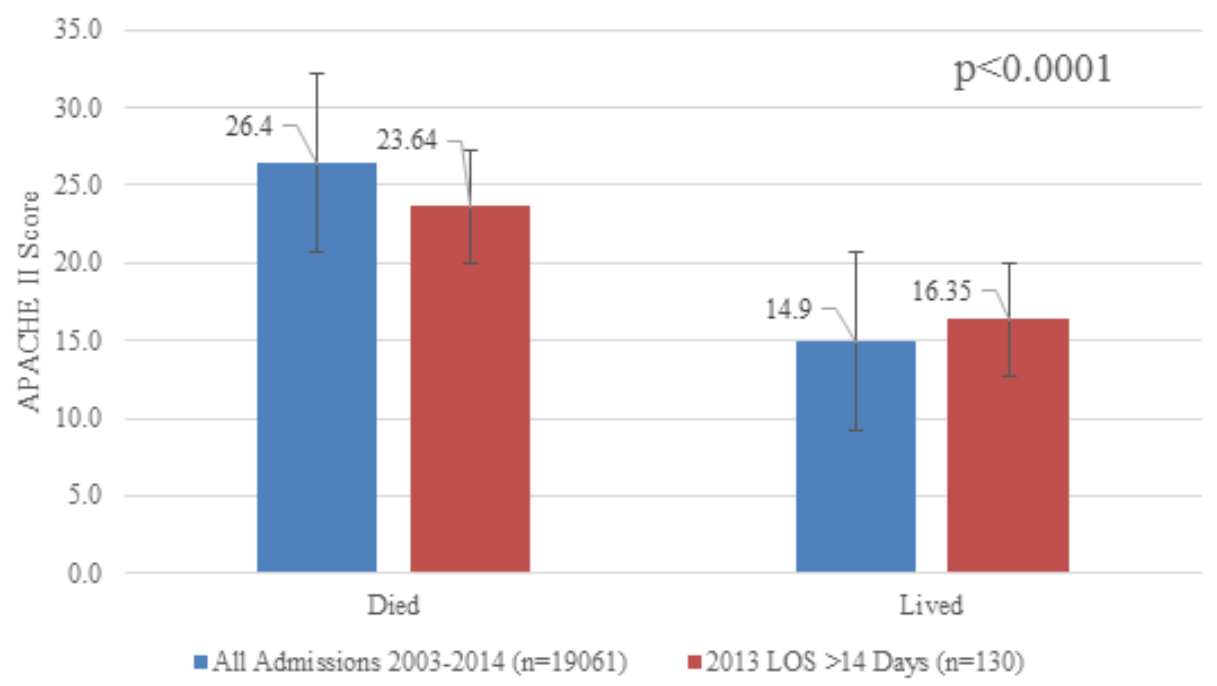

Figure 4. Average APACHE II scores on ICU admission and at day 14 of ICU stay further stratified by Mortality.

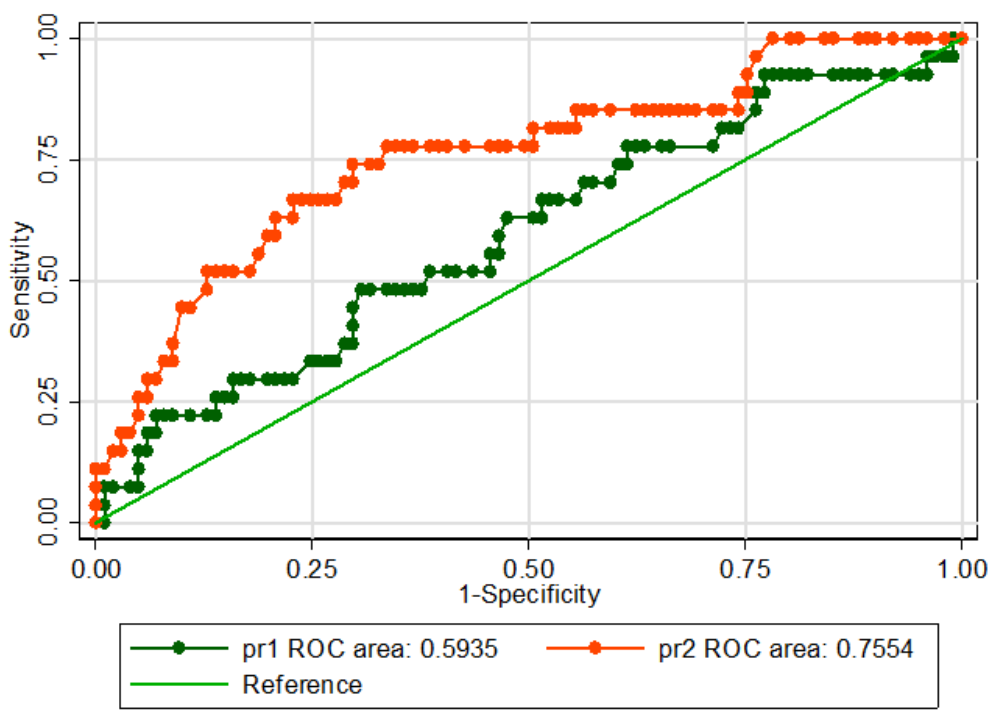

Figure 5. ROC curves for Admission and Day 14 Recalculated APACHE scores for the Prediction of Mortality. 
palliative care. This study represents the first of its kind surgical population assessment of the impact of ICU LOS on mortality. Although this relationship will likely vary based on institutional care patterns and patient populations, the method of determining the inflection point is applicable to all patient populations across all ICUs. Further, determining the inflection point denotes an important milestone during ICU care where enhanced communication and re-focused efforts may serve as a useful trigger for evaluating the appropriateness of the current goals of care.

We found an in-hospital mortality inflection point at day 14 , where mortality increased nearly 4 -fold. Previous definitions of a prolonged LOS broadly varied (5-60 days) with no clear consensus and without being grounded in universally applicable data (Table 1) [2,4,6-12]. Undoubtedly, this vast range reflects differences in patient population, practice pattern, and resource availability and utilization.

We further found that our best line of fit on the mortality vs. LOS plot maintained a relatively flat slope beyond the 2-week mark out through 6 weeks. This finding is similar to Williams et al who also observed an initially non-linear mortality relationship that plateaued after 10 days in an Australian mixed population $\mathrm{ICU}^{4}$. The mortality of the post-14-day ICU LOS population in our study was approximately $20 \%$. Nonetheless, while the observed mortality exceeds that of patients with shorter ICU LOS, we do not know if the mortality further increases beyond 6 weeks as that subset was not studied. Finally, long term functional outcome in survivors as well as family members of patients who survive prolonged critical illness are frequently compromised as has been shown in a multitude of recent studies. All of these findings should be considered when prognostication of outcome is performed.

Most illness severity and organ dysfunction scoring systems were designed for shorter ICU stays. Suistomaa et al. demonstrated a decrease in predictive value in customized versions of the Simplified Acute Physiology Score (SAPS) and APACHE beyond 7 days in an ICU [13]. Thus, contemporary use of APACHE II scoring is primarily used upon ICU admission. Our finding of direct mortality correlation of recalculated APACHE II scores on ICU day 14 adds a new dimension of predictive abilities in an ICU population. This is above and beyond the expected improved predictive value because the patients are theoretically 2 weeks closer to death. Our greater than 2 week cohort had an average survival well beyond two weeks, thus better validating the predictive findings that can guide appropriate decision making and therapeutic options.

Conversely, delayed assessment of mortality and LOS predictors has been described previously and have been found to be more sensitive and specific as compared to measures taken at the time of ICU admission. Kramer et al, in a refined predictive regression model of 343,555 ICU admissions identified that data collected on the fifth day proved to be highly predictive of a prolonged length of stay [6]. Further, they found that assessing predictors of prolonged LOS on day

\section{Day 14 Predictors of Mortality}

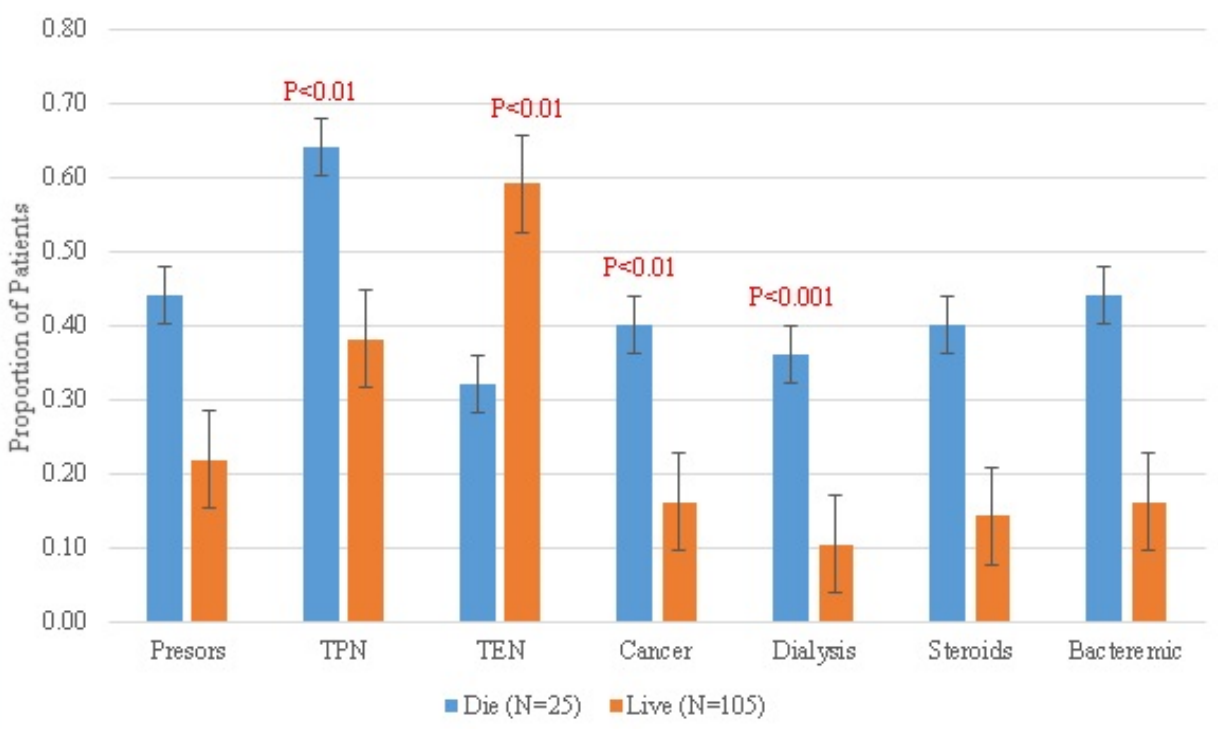

Figure 6. ICU day 14 predictors of mortality.

Table 1. Literature review for prolonged ICU length of stay.

\begin{tabular}{|c|c|c|c|c|c|}
\hline Author & Year & Prolonged Length of Stay & Defined by & Number of Patients & Type \\
\hline Arabi, Y. et. al [8] & 2002 & 14 days & Arbitrary cutoff & 6,392 & Single Institution \\
\hline Venker, J. et al [12] & 2005 & 60 days & Arbitrary cutoff & 12,021 & Single Institution \\
\hline Laupland, K.B. et al [2] & 2005 & 14 days & Arbitrary cutoff & 4,845 & Single Institution \\
\hline Friedrich, J.O. et. al [10] & 2006 & 30 days & Arbitrary cutoff & 3,172 & Single Institution \\
\hline Rimachi, R. et. al [11] & 2007 & 10 days & Mortality curve & 13,210 & Single Institution \\
\hline Albelha, F. et. al [7] & 2007 & 7 days & Arbitrary cutoff & 375 & Single Institution \\
\hline Ong, A.W. et al [9] & 2009 & 30 days & Arbitrary cutoff & 4,920 & Single Institution \\
\hline Williams, T.A. et. al [4] & 2010 & 10 days & Mortality curve & 22,298 & Single Institution \\
\hline Kramer, A.A. et al [6] & 2010 & 5 days & Upper quintile & 343,555 & 31 US Institutions \\
\hline Martin et al & 2016 & 14 days & Mortality curve & 19,061 & Single Institution \\
\hline
\end{tabular}


one of ICU admission reduced the accuracy of prediction because of the uncertainty of prognosis, subsequent complications, and variations in response to therapy [6].

Other predictors of mortality on the inflection point day included vasopressor infusion, TPN, steroids, dialysis, bacteremia, and cancer. Although not specifically investigated in this study, Ong et al also found that pre-existing renal disease and acute kidney injury requiring dialysis were independent predictors of mortality during a prolonged LOS [9]. Friedrich et al similarly found that with LOS greater than 30 days, the requirements for life support (as defined by needs for ventilation, vasoactive agents, or dialysis) or those with significant preexisting morbidities (such as immunosuppression and extremes of age) were at significant risk of mortality [10].

Thus, these factors, in conjunction with formalized scoring systems such as APACHE and SAPS may further assist assessing goals of care and directing decision making in patients who remain in a surgical ICU after the inflection point day. Extended use of scoring systems in this fashion has been previously described in the literature. Lissauer et al found predictive differences in ICU discharge profiles in patients who ultimately required re-admission verses those that did not using APACHE III, APACHE IV, and the Acute Physiology Score [14].

Beyond mortality, many of these indicators are also associated with an increased LOS independently and often have perform better as process-of-care metrics. Ong et al, again using 30 days as their definition for prolonged LOS, noted sepsis and ARDS contributed significantly [9]. More importantly, early predictors of prolonged LOS have been elucidated in several large cohort studies and have shown associations with SAPS survival probability, age 40-80 years, presence of infection or mechanical ventilation at 24 hours after admission, male gender, emergency surgery, trauma, presence of fellows, prolonged pre-ICU stays, severity of illness, presence of shock, and identification of bacteremia $[2,15]$. Although real-time assessment of these variables can be difficult to perform, as electronic health records become more versatile, data analysis and predictive scoring will become more readily available for prognostication [16].

This study has several potential limitations that should be noted. First, this is a single institution study and findings may represent unique organizational processes of care that are not necessarily reflective of other surgical ICUs. However, our methodology is readily applicable to any ICU population wishing to calculate their own intrinsic rate in order to initiate patient-centered outcomes. In many ways, the methods and discussion about recognizing this at-risk population is equally important to the statistical findings of this study that point to the 14 day point.

Next, the rationale for the specific predictors measured was based on the mortality predictors already identified in existing literature and was not inclusive of all available metrics. Additionally, we stopped data collection at 6 weeks and only captured in-hospital mortality (of note, our population very rarely used a "hospice" service that would have affected in-hospital mortality rates). One percent of our total population has a LOS greater than 6 weeks, and thus, the outcome of these patients is unknown. Finally, although all of our attention in this study is on mortality, many studies have documented significant morbidity (such as Post Intensive Care Syndrome that includes neuropathy and myopathy of critical illness, persistent inflammation, and immunosuppression syndrome) and long term (post discharge) mortality in initial survivors of critical illness and this should be taken into account during goals of care discussions [11,15,17-18].
The findings of this study are an important component of the growing body of end-of-life-care literature. Our proposed two-week time prompt can prevent practitioners from falling victim to treatment protocols and practices that can prevent them from astutely realizing futility or failure to improve. Discussing goals of care at a logical timepoint with all the stakeholders (the patient, family, consultants, primary teams, and the ICU team) can improve the value in healthcare delivery, minimizing unnecessary pain and suffering, all while specifically not increasing the overall mortality rate. One logical next step following this study would be to trigger a palliative care consultation at the two-week mark if other prognostic factors had not already triggered a consultation. Many studies have shown the efficacy of proactive palliative care interventions in other intensive care settings including a recent prospective study that found a shorter length of stay without any significant difference in mortality rate or discharge disposition $[19,20]$.

Finally, surgery patients in particular are at risk of delayed recognition of futile care secondary to the large upfront commitment by the surgeon. This upfront effort can temporarily dissuade the surgeon from realizing pending mortality and prolong the inevitable. Surgeons in particular then, be benefit from suggestions such as that from Curtis et al: to consider any treatment as a "time-limited trial and to prospectively consider the circumstances under which we should consider such a trial a failure-resulting in a transition to a focus on palliation" [21]. A "failure plan" can be shared amongst surgeons and families prior to surgery and could establish either an occurrence or a time point that warrants different considerations. This could include a pre-determined list of concrete post-operative complications predictive of poor outcomes.

This study has several limitations that should be noted. This is a retrospective, single institution study so its findings such as the day 14 inflection point may be variable at other institutions. However, as noted previously, the conceptual premise that there is an infection point still stands and this can be recalculated at any institution to assist with their own initiatives. Some factors that may affect mortality were not captured as part of this study such as discharges to hospice or Long Term Acute Care Hospitals (LTACHs) were mortality may still be realized. It should be noted that the surgical ICU utilized hospice nominally and LTACH transfers were not considered if long-term improvement was not expected. Finally, APACHE II scoring is not optimized to the surgical population as there is some lead time bias and chronic health evaluation points, but it is the system used by many institutions in ICU databases. This also accounts for the low ROC on admission seen in our data.

In conclusion, our study was able to analyze objective data and identify Day 14 as a critical time point at which in-hospital mortality changes. Further, at this time point, re-calculated APACHE II scores and other variables are predictive of mortality. Our methodology can further be used to critically evaluate locally relevant data in other ICU settings. In the future, prospective studies should be performed that empirically validate a model of proactive palliative care interventions in the SICU at pre-established time-points that reduce length of stay and cost without increasing mortality. This information can be used to develop distinct guidelines that allow physicians to predict prognosis at the appropriate time and better deliver optimal care.

\section{Author Contributions}

Literature search \& study design: Martin, Ramaswamy, Marks, Collins, Kaplan, O'Connor, Caserett, Reilly 
Data Collection: Martin, Collins, Marks, Holena, Pascual

Data interpretation: Ramaswamy, Holena, Pascual, Reilly, Kaplan, O'Connor, Caserett

Manuscript writing: Martin, Ramaswamy, Marks, O’Connor, Collins

Critical revision: Martin, Casarett, Holena, Pascual, Reilly, Kaplan

\section{Acknowledgement}

Patrick M. Reilly, MD was funded in-part, by NIH study: 5-R01NR-013503-04

\section{References}

1. Haas JS, Teixeira C, Cabral CR, Fleig AHD, Freitas APR, et al. (2013) Factors influencing physical functional status in intensive care unit survivors two years after discharge. BMC Anesthesiol 13: 11. [Crossref]

2. Laupland KB, Kirkpatrick AW, Kortbeek JB, Zuege DJ (2006) Long-term mortality outcome associated with prolonged admission to the ICU. Chest 129: 954-959. [Crossref]

3. Vest MT, Murphy TE, Araujo KL, Pisani MA (2011) Disability in activities of daily living, depression, and quality of life among older medical ICU survivors: a prospective cohort study. Health Qual Life Outcomes 9: 9. [Crossref]

4. Williams TA, Ho KM, Dobb GJ, Finn JC, Knuiman M, et al. (2010) Effect of length of stay in intensive care unit on hospital and long-term mortality of critically ill adult patients. Br J Anaesth 104: 459-464. [Crossref]

5. Wolters AE, van Dijk D, Pasma W, Cremer OL, Loiije MF, et al. (2014) Long-term outcome of delirium during intensive care unit stay in survivors of critical illness: a prospective cohort study. Crit Care 18: R125. [Crossref]

6. Kramer AA, Zimmerman JE (2010) A predictive model for the early identification of patients at risk for a prolonged intensive care unit length of stay. BMC Med Inform Decis Mak 10: 27. [Crossref]

7. Abelha F, Maia P, Landeiro N, Neves A, Barros H (2006) Determinants of Outcome in Patients Admitted to a Surgical Intensive Care Unit. Crit Care Med 34: p A139.

8. Arabi Y, Venkatesh S, Haddad S, Al Shimemeri A, Al Malik, S (2002) A Prospective Study of Prolonged Stay in the Intensive Care Unit: Predictors and Impact on Resource Utilization. Int J Qual Health Care 14: 403-410. [Crossref]
9. Ong AW, Omert LA, Vido D, Goodman BM, Protetch J, et al. (2009) Characteristics and outcomes of trauma patients with ICU lengths of stay 30 days and greater: a sevenyear retrospective study. Crit Care 13: R154. [Crossref]

10. Friedrich JO, Wilson G, Chant C (2006) Long-term outcomes and clinical predictors of hospital mortality in very long stay intensive care unit patients: a cohort study. Crit Care 10: R59. [Crossref]

11. Rimachi R, Vincent JL, Brimioulle S (2007) Survival and quality of life after prolonged intensive care unit stay. Anaesth Intensive Care 35: 62-67. [Crossref]

12. Venker J, Miedema M, Strack van Schijndel RJ, Girbes AR, Groeneveld AB (2005) Long-term outcome after 60 days of intensive care. Anaesthesia 60: 541-546. [Crossref]

13. Suistomaa M, Niskanen M, Kari A, Hynynen M, Takala J (2002) Customized prediction models based on APACHE II and SAPS II scores in patients with prolonged length of stay in the ICU. Intensive Care Med 28: 479-485. [Crossref]

14. Lissauer ME, Diaz JJ, Narayan M, Shah PK, Hanna NN (2013) Surgical intensive care unit admission variables predict subsequent readmission. Am Surg 79: 583-588. [Crossref]

15. Higgins TL, McGee WT, Steingrub JS, Rapoport J, Lemeshow S, et al. (2003) Early indicators of prolonged intensive care unit stay: impact of illness severity, physician staffing, and pre-intensive care unit length of stay. Crit Care Med 31: 45-51. [Crossref]

16. Pirracchio R, Petersen ML, Carone M, Rigon MR, Chevret S, et al. (2015) Mortality prediction in intensive care units with the Super ICU Learner Algorithm (SICULA): a population-based study. Lancet Respir Med 3: 42-52. [Crossref]

17. Livingston DH, Tripp T, Biggs C, Lavery RF (2009) A fate worse than death? Longterm outcome of trauma patients admitted to the surgical intensive care unit. J Trauma 67: 341-348. [Crossref]

18. Wunsch H, Guerra C, Barnato AE, Angus DC, Li G, et al. (2010) Three-year outcomes for Medicare beneficiaries who survive intensive care. JAMA 303: 849-856. [Crossref]

19. Ahrens T, Yancey V, Kollef M (2003) Improving family communications at the end of life: implications for length of stay in the intensive care unit and resource use. Am J Crit Care 12: 317-323. [Crossref]

20. Digwood G, Lustbader D, Pekmezaris R, Lesser ML, Walia R, et al. (2011) The impact of a palliative care unit on mortality rate and length of stay for medical intensive care unit patients. Palliat Support Care 9: 387-392. [Crossref]

21. Curtis JR, Engelberg RA, Bensink ME, Ramsey SD (2012) End-of-life care in the intensive care unit: can we simultaneously increase quality and reduce costs? Am J Respir Crit Care Med 186: 587-592. [Crossref]

Copyright: (C2018 Martin ND. This is an open-access article distributed under the terms of the Creative Commons Attribution License, which permits unrestricted use, distribution, and reproduction in any medium, provided the original author and source are credited. 